\title{
Functionalized carbon nanotubes as a filler for dielectric elastomer composites with improved actuation performance
}

\author{
Fabia Galantini $^{1}$, Sabrina Bianchi ${ }^{2}$, Valter Castelvetro ${ }^{2}$ and Giuseppe Gallone ${ }^{1,3}$ \\ 1 "E. Piaggio" Research Center, University of Pisa, Largo Lucio Lazzarino 1, 56122, Pisa, Italy \\ ${ }^{2}$ Department of Chemistry and Industrial Chemistry, University of Pisa, Via Risorgimento 35, 56122, \\ Pisa, Italy \\ ${ }^{3}$ Department of Civil and Industrial Engineering, Largo Lucio Lazzarino 1, 56122, Pisa, Italy \\ E-mail: fabia.galantini@ing.unipi.it
}

\begin{abstract}
Among the broad class of electro-active polymers, dielectric elastomer actuators represent a rapidly growing technology for electromechanical transduction. In order to further develop this applied science, the high driving voltages currently needed must be reduced. For this purpose, one of the most considered approach is based on making elastomeric composites with highly polarisable fillers in order to increase the dielectric constant while maintaining both low dielectric losses and high mechanical compliance. In this work, multi-wall carbon nanotubes were first functionalized by grafting either acrylonitrile or diurethane monoacrylate oligomers, and then dispersed into a polyurethane matrix to make dielectric elastomer composites. Procedures for the chemical functionalization of carbon nanotubes and proper characterizations of the obtained products are provided in detail. Consequences of the use of chemically modified carbon nanotubes as filler, in comparison to using unmodified ones, were studied in terms of dielectric, mechanical and electro-mechanical response. In particular, an increment of the dielectric constant was observed for all composites throughout the investigated frequency spectrum, but only in the cases of modified carbon nanotubes the loss factor remained almost unchanged with respect to the simple matrix, indicating that conductive percolation paths did not arise in such systems. An effective improvement in the actuation strain was observed for samples loaded with functionalized carbon nanotubes.
\end{abstract}

\section{Introduction}

The use of carbon nanotubes (CNTs) for polymers modification has recently opened new perspectives for multifunctional materials based on both thermoplastics [1], thermosettings [2], elastomers [3], bio-polymers [4] or liquid crystals [5]. Thanks to their nanometer-scale dimension, along with the large shape anisotropy, the high mechanical strength and the very high thermal and electrical conductivity, CNTs can induce significant changes in the material's properties even if they are added in low amount. So far, they have found application in fields like biosensors [6], composites [7], field emission devices [8], electronic components [9], probe tips [10], etc.

Combination of CNTs with electro-active polymers (EAPs), has recently found application not only as a reinforcing method to create structured materials with improved mechanical properties [11], but also as a means to enhance the electromechanical performance of dielectric elastomers actuators (DEAs) $[5,12,13,14]$. As a fact, the actuation strain achievable by a dielectric elastomer (DE) at fixed applied voltage can be written as [14]:

$s \sim\left(\mathcal{E}^{\prime} V^{2}\right) /\left(d^{2} Y\right)$

where $s$ is the strain generated by the electrostatic squeezing of a DE of thickness $d$ as a consequence of the application of an external voltage $V, \mathcal{E}^{\prime}$ and $Y$ being respectively the dielectric constant and the elastic modulus of the DE material. From Eq. 1, it can be easily recognized that, in order to improve the electromechanical response of a dielectric elastomer, it is necessary to enhance its dielectric constant while maintaining a fairly high mechanical compliance $[12,15]$. Moreover, the contemporary preservation of low dielectric losses is a not secondary constraint $[12,15]$. Thus, among the various approaches $[12,16,17,18,19]$ that have been proposed in order to improve the electromechanical performance of dielectric elastomers, 
CNTs can be considered a candidate for making elastomeric composites with improved actuation properties, due to their high polarisability.

Nevertheless, due to their tendency to aggregate and form clusters, CNTs have to be opportunely modified before they can be effectively used as a filler, in order to minimize side effects as agglomeration, percolation phenomena and dielectric strength reduction, and possibly to achieve better compatibility between matrix and filler. It is well known that non-covalent functionalization with surfactants of CNTs [20], as of other solids [21], can promote stable dispersions in different media. Such a methodology preserves the electrical properties of CNTs and, for this reason, it is preferred for electronic applications. Other approaches can be used to chemically treat CNTs and create covalent functionalization sites along the CNT external surface. They are widely described in literature and generally they consist in the creation of surface defects and subsequent functionalization from defects [22]. Most common CNTs functionalization methods include the use of diazonium [23], fluorination [24], radical chemistry [25], metal reduction [26] and others. Depending on the extended $\pi$-conjugation, many of the optical, electrical and thermal properties may become lost with covalent functionalization, but this can be turned as an advantage depending on the final application the material is designed for. In the field of DEAs, in particular, where nanotubes can be desirable just for their high polarisability, covalent functionalization is preferred since electrical percolation must be prevented in order to both allow for higher filler contents and preserve actuation capabilities at high electric fields.

Following these last considerations, the possibility of using a covalent functionalization process in order to obtain a filler for dielectric elastomer composites with enhanced electromechanical response is considered in this work. Procedures for the chemical functionalization of carbon nanotubes, together with proper characterisations of the obtained products, are provided in detail. Consequences of dispersing either pristine and functionalized CNTs into a dielectric elastomer are then presented and discussed. In particular, the effects of the chemical functionalization of CNTs on the elastomeric composites are analyzed in terms of those properties that drive the actuation response as from (1). As such, this work does not aim to be exhaustive, since some other relevant properties like tear resistance and dielectric breakdown threshold, which can be strongly affected by the addition of fillers, remain to be assessed with further studies. Nevertheless, this study provides a preliminary and necessary investigation in order to understand if the use of properly functionalized carbon nanotubes (FCNTs) may represent a viable route for obtaining new elastomeric composites suitable for applications as DEAs with enhanced performance.

\section{Materials and Methods}

Chemically modified CNTs were first obtained by proper functionalization of pristine multi-wall CNTs and then used as a filler into a commercial polyurethane based dielectric elastomer (PU in the following) to give elastomeric composites. Both fillers (CNTs and FCNTs) and composites were extensively characterized by techniques like infrared spectroscopy, thermogravimetry, transmission electron microscopy, dielectric spectroscopy, dynamic-mechanical and electromechanical analysis.

\subsection{CNTs functionalization}

Multi-wall CNTs, a gift from Bayer, were functionalized via Reversible Addition-Fragmentation chain Transfer (RAFT) grafting-from polymerization by using two different monomers: either acrylonitrile (AN) or a diurethane monoacrylate (H10). The grafting of CNTs was performed in a multi-step process. First, the dithioester RAFT agent ethoxycarboxyl-prop-2-yl dithiobenzoate was immobilized onto the surface of oxidized nanotubes (CNTs-RAFT) by a three-step synthetic procedure, as described in [27]. Then, the monomer was polymerized (AN $\rightarrow$ PAN; H10 $\rightarrow$ PH10) in presence of CNTs-RAFT, by using 2,2azobisisobutyronitrile (AIBN) as initiator. The whole reactions scheme is represented in figure 1. 


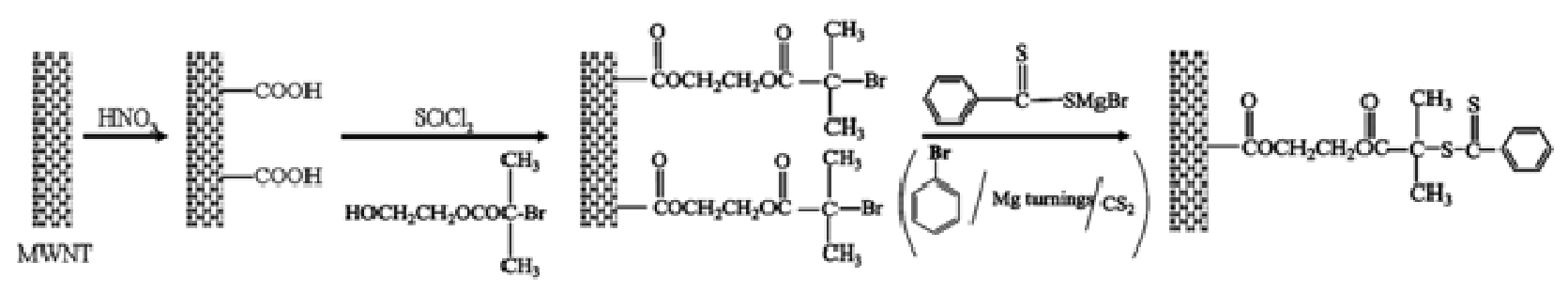

(1)

(2)

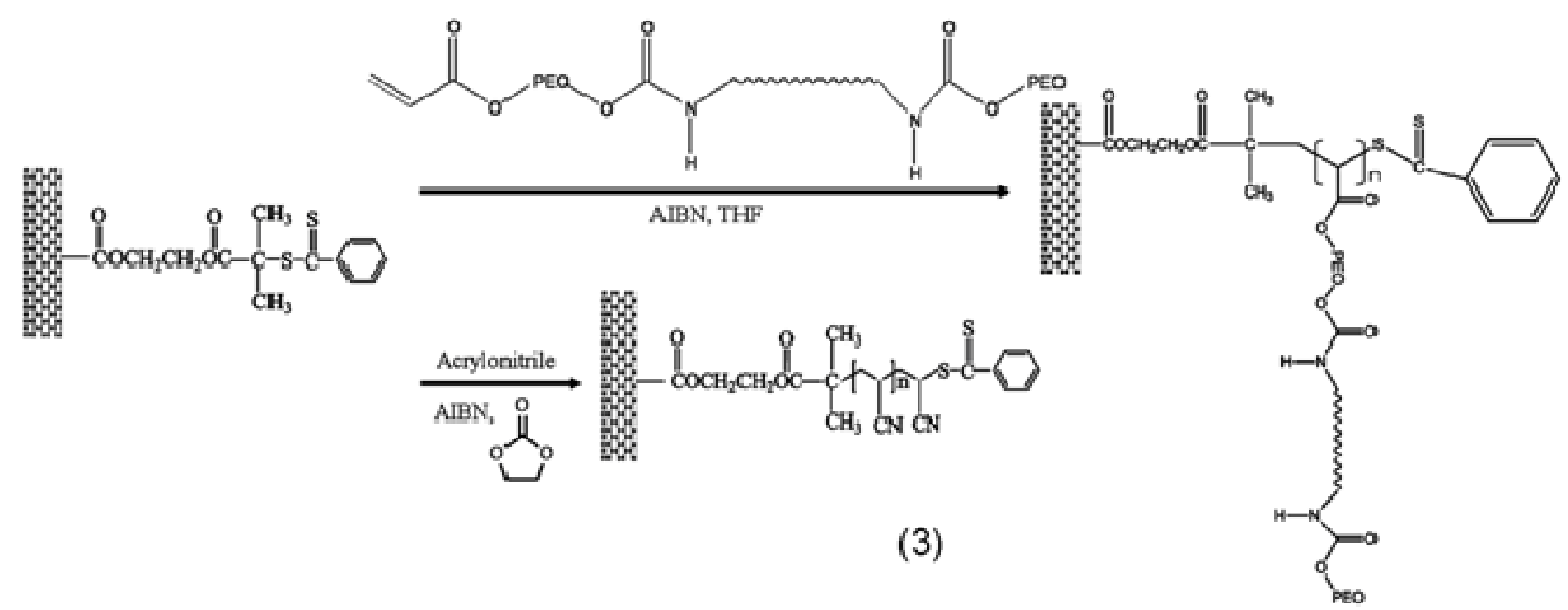

Figure 1. Chemical oxidation (1) and grafting of RAFT agent (2) onto CNTs.

Final RAFT grafting-from polymerization (3) of PH10 and PAN, respectively.

\subsection{Dielectric elastomer matrix and composites preparation}

A soft dielectric PU elastomer from Polytek, series Poly74-20 (hardness 20 ShoreA), was used as a matrix for the preparation of high permittivity elastomeric composites. It is commercially supplied in the form of two liquid reactants, A (diisocyanates) and B (polyols), which must be mixed together in a 1:2 weight ratio and left resting for $48 \mathrm{~h}$ at room temperature to cure, as from supplier recipe. Composites between elastomeric PU and nanotubes (either pristine CNTs or FCNTs, both in the form of powder) were prepared by first dispersing the nanotubes in $\mathrm{CHCl}_{3}$ at ambient temperature and then by adding the obtained suspension to the PU reaction mixture. Samples were finally obtained by casting the reaction mixture into a glass Petri dish and leaving it at rest at ambient temperature for at least two days, in order to allow both matrix curing and solvent evaporation. Filler loading factors of 0.5 and $1 \mathrm{wt} \%$ were studied for CNTs functionalized with PH10 (CNTs-g-PH10) while a $5 \mathrm{wt} \%$ was tested for CNTs functionalized with PAN (CNTs-g-PAN). For comparison purposes, samples containing $1 \mathrm{wt} \%$ of CNTs-g-PH10 were also prepared without using $\mathrm{CHCl}_{3}$ as suspension medium, and in the following they are referred as "dry" type.

\subsection{Infrared spectroscopy}

Attenuated total reflectance (ATR)-IR spectra were collected on a Perkin-Elmer spectrometer mod. 1760-X with PIKE MIRacle ATR accessory. Intermediate reactants and final products were analyzed in order to verify the success of the different step-reactions.

\subsection{Thermogravimetry}

Thermogravimetric (TGA) analyses were carried out on a Mettler Toledo TGA/SDTA 851 apparatus, with nitrogen gas as the purge gas $(60 \mathrm{~mL} / \mathrm{min})$ and at a heating rate of $10^{\circ} \mathrm{C} / \mathrm{min}$, from 25 to $600{ }^{\circ} \mathrm{C}$. Both CNTs-RAFT and FCNTs were analyzed, and the amount of grafted polymer was estimated.

\subsection{Transmission electron microscopy}

A Philips CM12 transmission electron microscope was used for morphological characterization of carbon nanotubes. It is a general purpose analytical transmission/scanning transmission electron microscope 
(TEM/STEM) equipped with thermo-ionic electron source, whose beam acceleration voltage can be selected in a range 20 to $120 \mathrm{kV}$. Image resolution can be better than $0.34 \mathrm{~nm}$ for point to point method, and $0.2 \mathrm{~nm}$ for line to line method. Magnification range can be varied from $31 \times$ to $66 \cdot 10^{4} \times$. High resolution images of both pristine and functionalized carbon nanotubes were acquired from drops of CNTs/chloroform dilute solutions deposited on a $2 \times 2 \mathrm{~mm} \mathrm{Cu} / \mathrm{C}$ grid. Images were analyzed by means of ImageTool image processing and analysis software by the University of Texas Health Science Center (San Antonio, Texas, USA), in order to make an estimation of the thickness of the functionalization layer.

\subsection{Dielectric spectroscopy}

A vector network analyzer (ZVRE from Rohde \& Schwartz) was used [17] to study the dielectric response of both pure matrix and composites. Spectra of the real and imaginary parts of the complex dielectric permittivity $\mathcal{E}^{*}$ (see (2)) were measured in the frequency range $\mathrm{f}=2 \pi \omega=10 \div 10^{8} \mathrm{~Hz}$ by testing disk shaped specimens of $11 \mathrm{~mm}$ diameter and less than $1 \mathrm{~mm}$ thick.

$\varepsilon^{*}(\omega)=\varepsilon^{\prime}(\omega)-j \varepsilon^{\prime \prime}(\omega)$

\subsection{Dynamic-mechanical analysis}

DMA was carried out by means of a dynamometer mod. Eplexor $100 \mathrm{~N}$ by Gabo Qualimeter Testanlagen $\mathrm{GmbH}$, on rectangular strips $(20 \times 10 \times 1 \mathrm{~mm})$ specimens. Elongation tests were performed at ambient temperature. Dynamic strains of 5\% amplitude, superimposed to a static pre-strain of $10 \%$, were applied to the samples at various frequencies in the range $0.1 \div 100 \mathrm{~Hz}$, and the dynamic-mechanical properties were studied in terms of the complex elastic modulus $Y^{*}(3)$.

$Y^{*}(\omega)=Y^{\prime}(\omega)+j Y^{\prime \prime}(\omega)$

\subsection{Electromechanical analysis}

Electromechanical actuation tests were carried out on properly prepared specimens of both pure matrix and composites. For every material sample, small strips of about $30 \times 10 \times 1 \mathrm{~mm}$ were cut from a film prepared by casting as described above. Electrically conductive carbon grease electrodes (Nyogel 755G Tecnolube Seal, USA) were then smeared on both the greater faces, leaving a $1 \mathrm{~mm}$ frame along the strip borders free of grease, in order to avoid possible electrical shorts and arching between the two oppositely charged sides. Electrical contacts to connect a high-voltage power supply (HV-DC 205A-30P, Bertan, USA) to the carbon grease electrodes were obtained through two small bands of conductive adhesive tape. Each specimen was vertically arranged by clamping its top end to a rigid support, while a proper weight was attached to the bottom end in order to obtain a passive vertical pre-strain of $10 \%$. Actuation tests were performed by applying step-wise increasing voltages to the specimens along their thickness. For each voltage, the steadystate transverse strain of the material along its major (vertical) dimension was measured by using an Halleffect isotonic transducer (Mod. 7006, by Ugo Basile) tied to the bottom end of the sample.

\section{Results and Discussion}

Graphs reported in figure 2 show results from IR analysis. For CNTs-RAFT the spectrum appears quite flat, which is typical for purely graphitic structures, with an absorption peak at $1560 \mathrm{~cm}^{-1}$ relative to $\mathrm{C}=\mathrm{C}$ bonds stretching of graphene structures and two other peaks respectively due to the stretching vibrations of $\mathrm{C}=\mathrm{S}$ bonds $\left(1167 \mathrm{~cm}^{-1}\right)$ and to the out-of-plane deformation vibrations in the aromatic rings $\left(754 \mathrm{~cm}^{-1}\right)$, both from RAFT dithiobenzoate. 


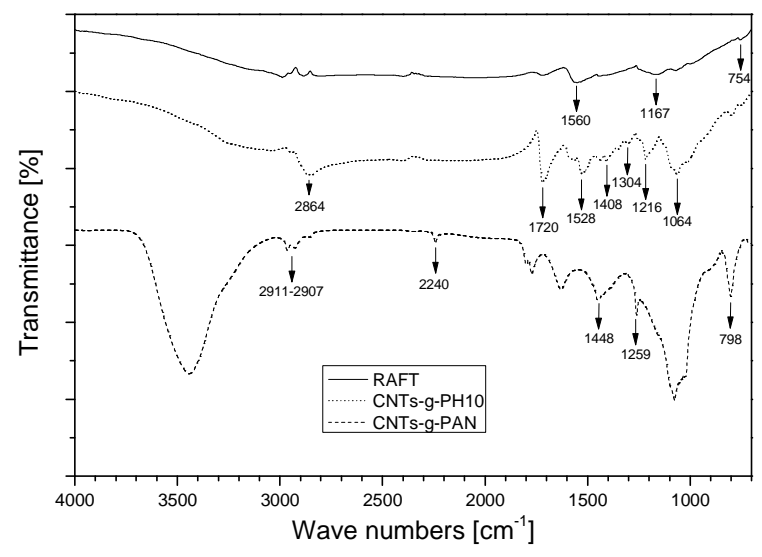

Figure 2. ATR-IR qualitative spectra of CNTs-RAFT, CNTs-g-PH10 and CNTs-g-PAN.

CNTs-g-PH10 spectrum exhibits more resolved absorption peaks, among which those relative to the $\mathrm{C}=\mathrm{O}$ $\left(1720 \mathrm{~cm}^{-1}\right)$ and C-O-C $\left(1064 \mathrm{~cm}^{-1}\right)$ stretching are visible and clear, confirming the presence of $\mathrm{H} 10$ carboxylic and ether groups. Finally, IR spectrum of the CNTs-g-PAN shows absorption peaks due to C $\equiv N$ $\left(2240 \mathrm{~cm}^{-1}\right)$ stretching in PAN, along with peaks from C-O-C stretching $\left(1259 \mathrm{~cm}^{-1}\right)$ and deformation (798 $\left.\mathrm{cm}^{-1}\right)$, and from various $\mathrm{C}-\mathrm{H}$ types of vibrations $\left(1448 \mathrm{~cm}^{-1}\right)$. By means of the thermograms in figure 3 , the final yield of the functionalization process can be estimated in terms of weight enhancement due to the grafted polymers, which results to be about $10 \mathrm{wt} \%$ for CNTs-g-PH10 and about $30 \mathrm{wt} \%$ for CNTs-g-PAN, as it comes out from the differences in weight loss at $500{ }^{\circ} \mathrm{C}$ with respect to CNTs-RAFT.

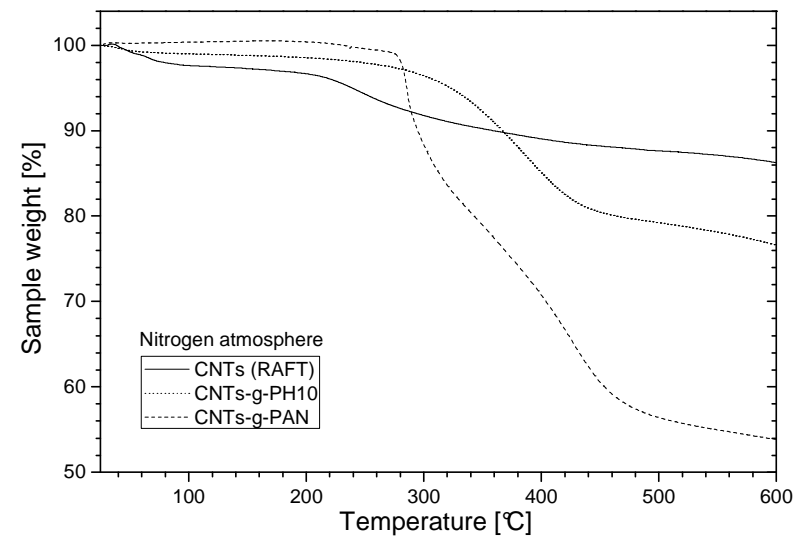

Figure 3. Thermograms of CNTs-RAFT and FCNTs.

In figure 4 are reported some TEM images for both unmodified and functionalized CNTs, which show a typical hollow and multi-walled structure for all the nanotubes. Moreover, by comparing images from both CNTs-g-PH10 and CNTs-g-PAN with respect to pristine CNTs, it is clearly recognizable the presence of an additional external layer in the functionalized CNTs, which is not found in the unmodified ones. 

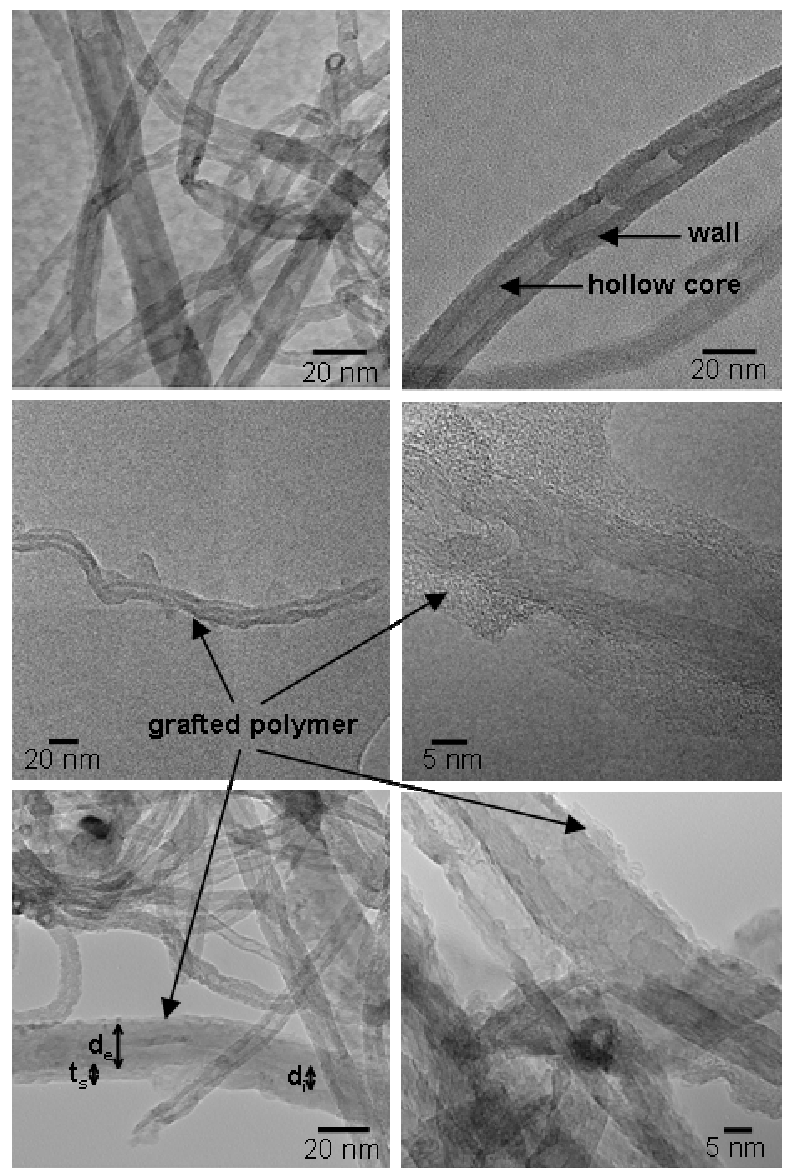

Figure 4. TEM images of pristine CNTs (upper row), CNTs-g-PAN (middle row) and CNTs-g-PH10 (bottom row) with indication of internal diameter $\left(d_{\mathrm{i}}\right)$, external diameter $\left(d_{\mathrm{e}}\right)$ and sheath thickness $\left(t_{\mathrm{s}}\right)$.

Such kind of sheath, which also makes the functionalized CNTs thicker than pristine CNTs, is very likely to be identifiable with the polymeric coating resulting from the functionalization process. From the TEM images, both internal, $d_{i}$, and external, $d_{e}$, diameters of bare nanotubes, together with the thickness, $t_{s}$, of the grafted polymer layer (see figure 4 for clarification) were measured and averaged over about ten pristine and CNTs-g-PH10. Results are shown in Table 1.

Table 1. Average values (nm) of $d_{\mathrm{i}}, d_{\mathrm{e}}$ and $t_{\mathrm{s}}$ for a sample of about ten specimens.

\begin{tabular}{cccc}
\hline & $\left\langle d_{i}\right\rangle$ & $\left\langle d_{e}\right\rangle$ & $\left\langle t_{s}\right\rangle$ \\
\hline CNTs & $6.3 \pm 2.4$ & $14.8 \pm 3.9$ & n.a. \\
\hline CNTs-g-PH10 & $3.5 \pm 1.3$ & $13.3 \pm 3.6$ & $2.4 \pm 1$ \\
\hline
\end{tabular}

Although the available statistics did not allow for an accurate estimation of the involved lengths, it is possible to notice a good agreement of the $d_{e}$ values coming from CNTs-g-PH10 and pristine CNTs, whereas the aforementioned additional thickness can be well characterized in CNTs-g-PH10. On the other end, the reduced average internal diameter observed for CNTs-g-PH10, with respect to pristine CNTs, could be ascribed to both a grater intrinsic variability of this quantity, being the carbon nanotubes multi-walled, and to a major difficulty to clearly distinguish the inner walls of the functionalized CNTs. In fact, it must be considered that the presence of a polymeric coating on a functionalized CNT surface is expected to appear, in the relative TEM image, not only as an additional layer at the borders profile, but also as a "visual noise" superimposed to the image of the nanotubes core (figure 4).

In figure 5, the sheath thickness for CNTs-g-PH10 is reported versus the external carbon nanotube diameter. The observed trend, moderately increasing with the CNTs surface size, accounts for an increased accessibility to the reactive sites on the nanotubes surface for the attaching polymer. Thus, as the nanotubes diameter grows, possible steric hindrance effects between adjacent active sites are lowered, and this allows grafting of longer molecular chains. 


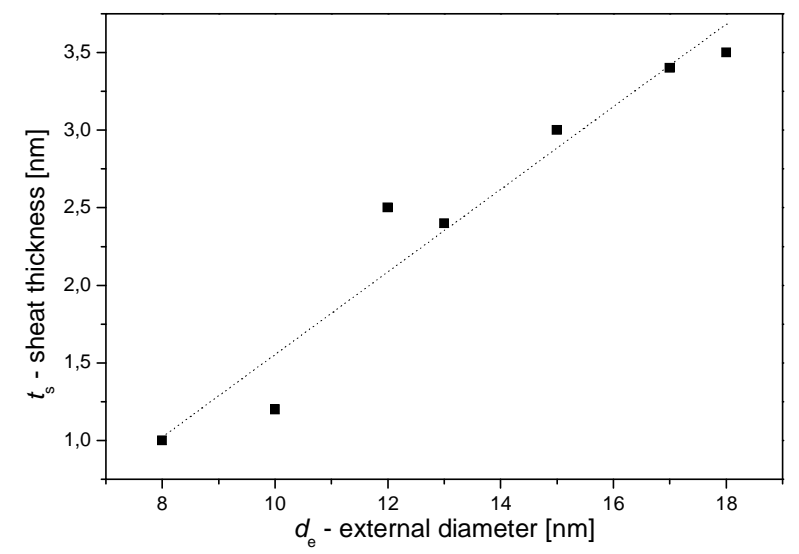

Figure 5. Sheath thickness $v s$ external diameter (dotted line is a guide for the eye).

Once verified that the functionalization process had been successful, pristine CNTs and FCNTs were added to the elastomeric matrix as described previously, and the obtained materials were dielectrically, mechanically and electromechanically analyzed. After matrix curing, composites showed different morphologies depending on both the amount and the nature of the used filler (figure 6). Surface of PU composites with pristine CNTs was rough (figure 6b), that of composites made by using CNTs-g-PAN was dappled (figure 6c), whereas for composites with CNTs-g-PH10 (figure 6d, 6e) it appeared smooth as for pure PU (figure 6a). This was probably due to a more effective compatibilization between filler and matrix resulting from the chemical functionalization with PH10. The use of a solvent allowed also a better dispersion of CNTs-g-PH10 into the matrix, as it is recognizable after comparing figures $6 \mathrm{~d}$ and $6 \mathrm{e}$. Indeed, samples prepared without chloroform solvent resulted in a less homogeneous, coarse-grained dispersion of filler.
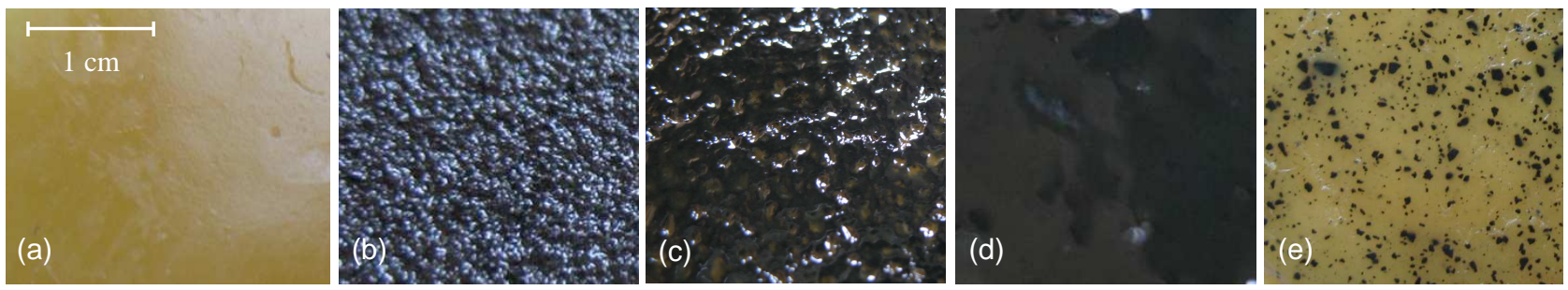

Figure 6. Images of pure PU (a) and PU loaded with 1 wt\% CNTs (b), 5 wt \% CNTs-g-PAN (c), $1 \mathrm{wt} \%$ CNTs-g-PH10 (d) and $1 \mathrm{wt} \%$ CNTs-g-PH10 dry (e).

Dielectric constants of both composites and pure matrix, which are all shown in figure 7a, were very different in the two cases of composites made with pristine CNTs and FCNTs. The consequences of dispersing functionalized carbon nanotubes are more clearly visible in the magnification of figure $7 \mathrm{~b}$, where the spectra of all the FCNTs loaded samples and of the simple matrix appear to have a similar pattern. These spectra show a little contribution from interfacial polarization, which causes the rising of the dielectric constant values as the frequencies decrease under $300 \mathrm{~Hz}$, followed by a smoothly dispersive, almost flat, profile at intermediate frequencies till about $10 \mathrm{MHz}$. A final more pronounced dispersive behavior is then observed at the highest frequencies. 

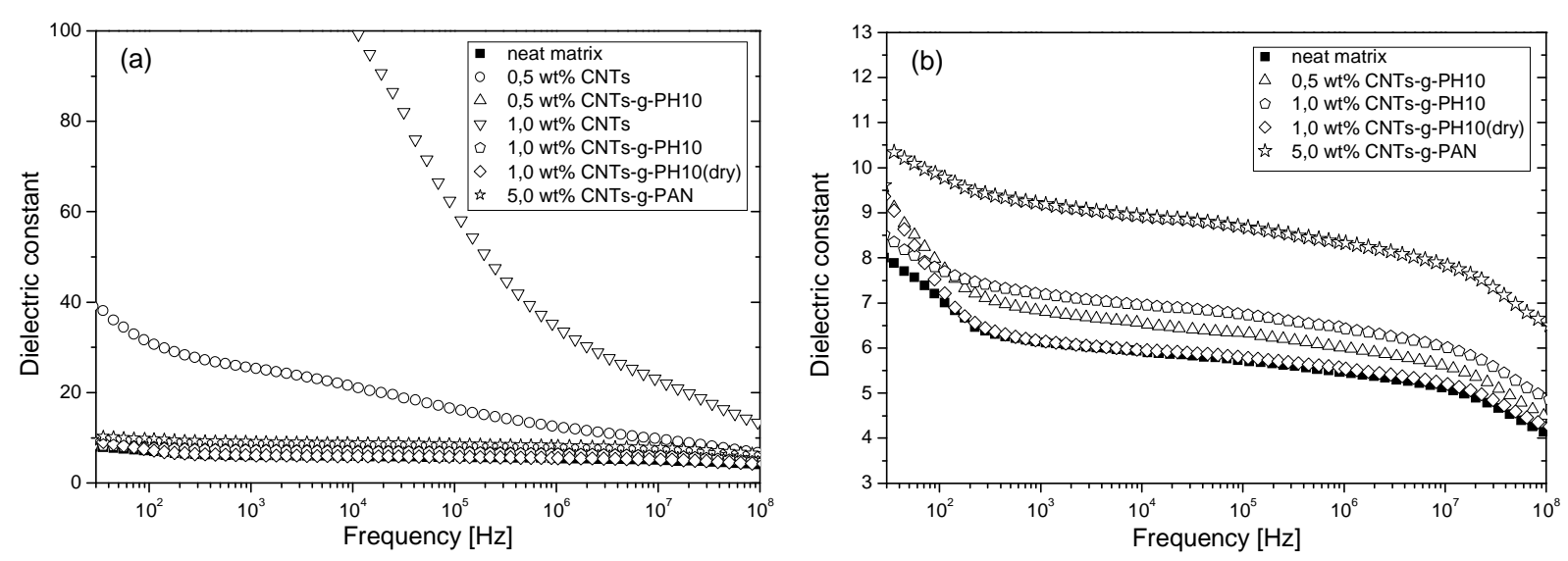

Figure 7. Spectra of the real part (dielectric constant) of the dielectric permittivity for elastomeric composites (a). In (b), a magnification for better comparison of FCNTs loaded systems.

Apart from a slight variability in the interfacial polarization effects, which at such low extents might not be very significant, an effective increment of the dielectric constant was observed throughout the remaining spectrum $\left(10^{2}-10^{8} \mathrm{~Hz}\right)$ of the samples loaded with CNTs-g-PH10 and CNTs-g-PAN, compared to the simple PU matrix. Evidently due to an increase of the bulk polarization, whose contribution dominates in the intermediate frequencies region, the dielectric constant reached a value of about 9 at $10 \mathrm{kHz}$ for the $5 \mathrm{wt} \%$ CNTs-g-PAN composite, compared to the value of about 6 observed for the pure matrix at the same frequency. However, the increasing effect on the dielectric constant of the pure matrix resulted to be monotonically dependent on the filler content, except for the case of sample containing $1 \mathrm{wt} \% \mathrm{CNTs-g-}$ PH10(dry). This last, which was prepared without the use of a solvent, showed approximately the same dielectric constant and dielectric loss (see figure 8a) of the pure matrix, apart from a slight increment in the dielectric constant at frequencies below $100 \mathrm{~Hz}$, due to the arising of an interfacial polarization contribution. This suggests that an inhomogeneous dispersion of the filler, as seen in figure 6e, can result less effective in increasing the overall polarisability of the material with respect to a finer and more homogeneous distribution, which instead ensures an increase of the bulk dielectric constant throughout the whole spectrum.

The addition of neat CNTs caused a dramatic increase of the dielectric constant throughout the investigated frequency range, both at $0.5 \mathrm{wt} \%$ and at $1 \mathrm{wt} \%$ loading factors (see figure $7 \mathrm{a}$ ). Nevertheless, pristine CNTs were also responsible of a very large increase of the dielectric loss, which instead remained almost unchanged after addition of FCNTs to the PU matrix (see figure 8a), indicating that percolation phenomena did not occur in systems loaded with FCNTs. In effect, composites made with not functionalized CNTs showed a substantial conductive electrical response, clearly recognizable in the $\omega^{-1}$ dependence of the dielectric loss $\mathcal{E}^{\prime \prime}$ at low frequencies. The calculated electrical conductivity for some tested formulations is reported in figure $8 \mathrm{~b}$ as a function of filler content. It appears that the conduction properties of composites made with neat CNTs had a dependence from the filler content resembling a typical percolative behavior. Such a behavior, instead, was not observed in composites made with CNTs-g-PH10, indicating that the grafted organic component played an effective role of electrically insulating sheath over the carbon nanotubes surfaces. This last finding is very desirable, since in this way the isolation properties of the dielectric elastomer, and thus its dielectric strength, are preserved. Indeed, the huge dissipative response observed in CNTs composites caused the impossibility of performing further electromechanical tests on such composites. 

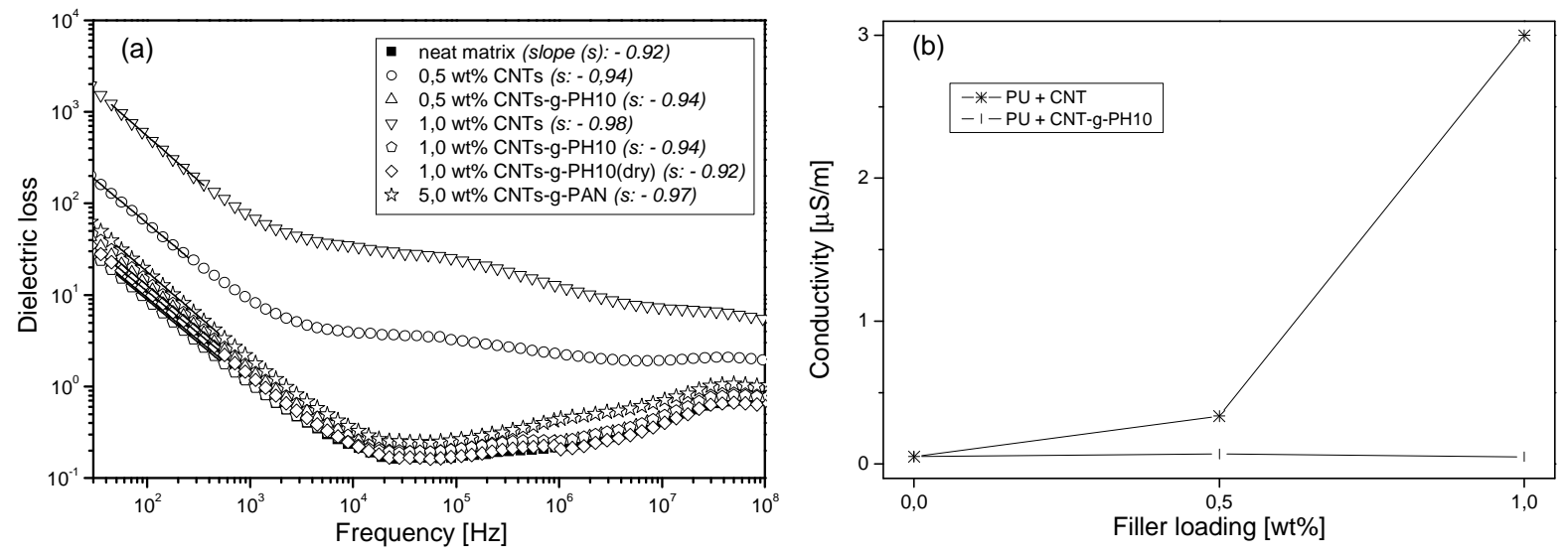

Figure 8. (a) Dielectric loss spectra for different composites. (b) Conductivity values at $60 \mathrm{~Hz}$ for PU samples loaded with different amount of pristine and FCNTs (solid lines are guides for the eyes).

From dynamic-mechanical tests carried out on both pure matrix and composites (figure 9), it emerged that all composites were softer than the PU matrix, especially samples containing FCNTs. As it has been also observed for other composite systems, a lower elastic modulus $Y^{\prime}$ could either be indicative of cavitation around the filler particles, favoured by a lack of adhesion between matrix and particles [28, 29], or be ascribed to a partial inhibition of the matrix curing process [30].

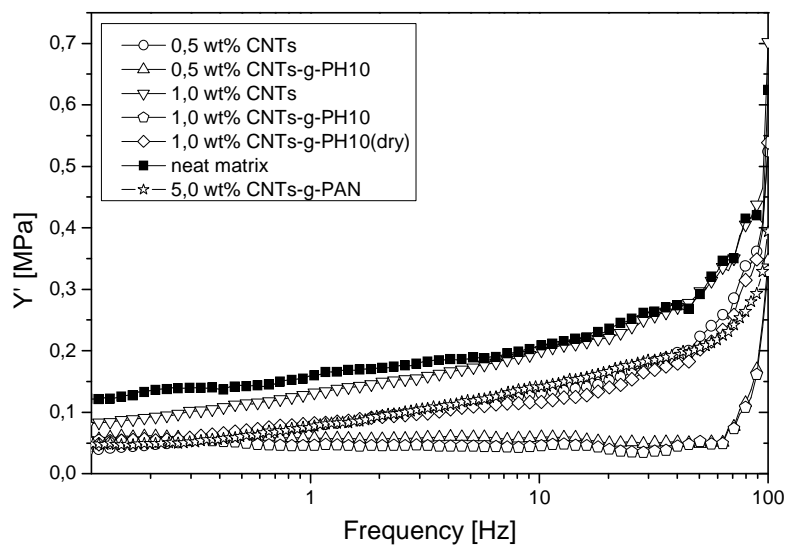

Figure 9. Elastic modulus of PU composites at room temperature, $10 \%$ static strain and $2 \%$ dynamic strain for frequency ranging from 0 to $100 \mathrm{~Hz}$.

Considering the very low level $(10 \pm 5 \%)$ of the strain applied during the tests, in this case the observed decrease of the elastic modulus is more likely to be attributed to a competing activity of the filler surface with respect to the chemical mechanism of the PU matrix curing process. Moreover, the fact that $1 \mathrm{wt} \%$ CNTs-g-PH10(dry) composites resulted stiffer than the $1 \mathrm{wt} \%$ CNTs-g-PH10 ones gives further support to this frame of interpretation, since a coarse-grained distribution like that of the "dry" filler (figure 6e) is expected to produce a less extended overall interface with the matrix, compared to the finer and homogeneous filler distribution of figure $6 \mathrm{~d}$. Indeed, a less extended matrix/filler interface entails a reduction of any chemical and physical interaction, and appears consistent with a parallel reduction of any possible competing activity of the filler with the crosslinking process. As a consequence, the observed stiffening of CNTs-g-PH10(dry) sample with respect to the CNTs-g-PH10 one can be ascribed to a higher curing density for the "dry" composite.

However, for frequencies greater than $0.5 \mathrm{~Hz}$ the softening effect was less evident in the case of pristine CNTs with respect to FCNTs at the same filler loading factor. This suggests that PH10 and PAN external sheaths were more effective in reacting with the PU matrix reactants than the bare CNT surface.

In the light of the aforementioned considerations, it is also possible to give an overall picture of the trends observed in figure 9 with respect to different loading factors for the same filler. Almost throughout the entire spectrum, the lowest stiffness level was reached by 0.5 and $1 \mathrm{wt} \%$ CNTs-g-PH10 composites, which showed $Y^{\prime}$ values very close from each other and only slightly increasing with the loading factor. This 
suggest that, for this filler, the chemical interference of the functionalized surface with the crosslinking process was very effective. After a certain extent, however, further additions of a filler like CNT, which is much stiffer than the matrix, caused the bulk effects to start becoming competitive or even predominant in determining the composite mechanical properties. Similar considerations can be made when comparing the 0.5 and $1 \mathrm{wt} \%$ pristine CNTs composites. This time, however, the filler surface turned out to be chemically less active, as already noted before, and the bulk effects of the filler addition resulted in a much higher stiffness of $1 \mathrm{wt} \%$ CNT composite with respect the $0.5 \mathrm{wt} \%$ CNT one.

The relevant dielectric and mechanical properties of $\mathrm{PU}$ matrix and composites at $50 \mathrm{~Hz}$ are reported in table 2 for direct comparison, along with the resulting $\mathcal{E}^{\prime} / Y^{\prime}$ ratios. Thanks to their lower elastic moduli and their enhanced dielectric constants, all composites showed $\varepsilon^{\prime} / Y^{\prime}$ ratios higher than that of PU matrix, which in the light of (1) means higher expected actuation performance.

Table 2. Dielectric $\left(\mathcal{\varepsilon}^{\prime}, \mathcal{E}^{\prime}\right)$ and mechanical $\left(Y^{\prime}\right)$ properties values and $\mathcal{\varepsilon}^{\prime} / Y^{\prime}$ ratio for samples tested at $50 \mathrm{~Hz}$.

\begin{tabular}{lcccc}
\hline Sample & $\boldsymbol{\varepsilon}^{\prime}$ & $\boldsymbol{\varepsilon}^{\prime \prime}$ & $\begin{array}{c}\boldsymbol{Y}^{\prime} \\
(\mathrm{kPa})\end{array}$ & $\begin{array}{c}\boldsymbol{\varepsilon}^{\prime} / \mathbf{Y}^{\prime} \\
\left(\mathrm{kPa}^{-1}\right)\end{array}$ \\
\hline Neat matrix & 7.6 & 17.6 & 292 & 0.026 \\
\hline $0.5 \mathrm{wt} \%$ CNTs-g-PH10 & 8.6 & 24.4 & 48 & 0.18 \\
$0.5 \mathrm{wt} \%$ CNTs & 35 & 115 & 223 & 0.16 \\
\hline 1wt\% CNTs-g-PH10 & 8 & 17 & 52 & 0.15 \\
1wt\% CNTs & $\sim 200$ & 1088 & 297 & $\sim 0.67$ \\
1wt\% CNTs-g-PH10(dry) & 8.4 & 2.5 & 202 & 0.04 \\
\hline 5 wt\% CNTs-g-PAN & 10.2 & 35 & 205 & 0.05 \\
\hline
\end{tabular}

However, despite the fact that the $1 \mathrm{wt} \%$ CNT filled composite exhibited the highest $\varepsilon^{\prime} / Y^{\prime}$ ratio, it did not show the best electromechanical response. As a fact, both composites containing 0.5 and $1 \mathrm{wt} \%$ of pristine CNTs resulted too lossy to actuate: they could not retain any charge distribution at their electrodes during actuation tests and substantial current flows were detected even at rather limited applied voltages (I > 500 $\mu \mathrm{A}$ at $\Delta \mathrm{V}>500 \mathrm{~V})$. Conversely, since dielectric losses for every composite made with FCNTs were extremely low, they allowed for successful electromechanical actuation tests. As a remarkable result, samples containing $0.5 \mathrm{wt} \%$ CNTs-g-PH10 showed an appreciably improved electromechanical response (figure 10), having provided about a $0.8 \%$ of actuation strain that doubled the $0.4 \%$ obtained from the matrix at the same applied electric field of $5 \mathrm{~V} / \mu \mathrm{m}$. Indeed, the $0.5 \mathrm{wt} \% \mathrm{CNTs}-\mathrm{g}-\mathrm{PH} 10$ was not only the best performing material in the actuation tests, but it resulted also the one having the highest $\varepsilon^{\prime} / Y^{\prime}$ ratio between the studied samples, apart from the non-actuating $1 \mathrm{wt} \%$ CNT composite (see table 2).

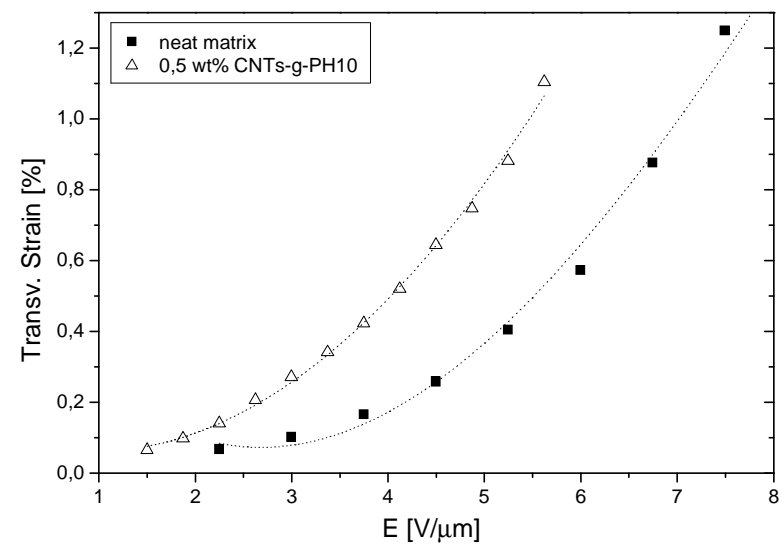

Figure 10. Electromechanical strain response exhibited by samples at $10 \%$ passive pre-strain (dotted lines are as a guide for the eye).

It is worth noting that these results confirm the key role of the $\mathcal{E}^{\prime} / Y^{\prime}$ ratio on the actuation properties, as prescribed by equation 1 . Nevertheless, in order to account also for the behaviour exhibited by both composites with bare CNTs, it must be recognized that the role played by both dielectric constant and elastic modulus is not exclusive, and that also the necessity of maintaining sufficiently low losses is of paramount importance. In this light, the choice to functionalize the CNTs before using them as a filler was proven to be 
successful for three reasons. In fact, in this way it was possible both to enhance the dielectric constant, by adding highly polarisable CNTs, to reduce the elastic modulus, due to the chemical action exerted by the functional groups grafted to CNTs, and to preserve low electric losses, in consequence of the electrically insulating polymeric sheath surrounding each conductive filler particle.

\section{Conclusions}

A procedures for the successful chemical functionalization of carbon nanotubes was presented, by means of which multi-walled carbon nanotubes were modified by grafting with either a poly-acrylonitrile or a diurethane polyacrylate. The obtained FCNTs were added to a dielectric elastomer matrix in order to make elastomeric composites and test their capability of enhancing the actuation response of the starting EAP. A significant increase in the dielectric constant and a reduction of elastic modulus were observed for composites containing 0.5 and $1 \mathrm{wt} \%$ CNTs-g-PH10 and $5 \mathrm{wt} \%$ CNTs-g-PAN with respect to the PU matrix. All the formulations loaded with FCNTs were found able to actuate. In particular, due to its best combination of dielectric and mechanical properties among all the studied materials, the elastomer loaded with a $0.5 \mathrm{wt} \%$ CNTs grafted with the diurethane polyacrylate, showed an electromechanical response which doubled that of the starting matrix. Moreover, functionalization proved to be effective in avoiding percolative effects in the electrical conductivity of the FCNTs filled composites, while composites containing pristine CNTs were electrically conductive and, as such, not capable of actuation at all.

\section{Aknowledgements}

The authors thanks Prof. M. De Sanctis and Dr. R. Ishak from the Department of Civil and Industrial Engineering, University of Pisa, for having kindly provided some of the TEM images reported in this work.

The "Fondazione Cassa di Risparmio di Pisa" is also gratefully acknowledged for the financial support 'POLOPTEL' project n. 167/09.

\section{References}

[1] Potschke P, Dudkin S M and Alig I 2003 Dielectric spectroscopy on melt processed polycarbonatemultiwalled carbon naotube composites Polymer 44 5023-5030

[2] Kim B, Lee J and Yu I 2003 Electrical properties of single-walled carbon nanotube and epoxy composites J. Appl. Phys. 94 6724-6728

[3] Cantournet S, Boyce M C and Tsou A H 2007 Micromechanics and macromechanics of carbon nanotube-enhanced elastomers J. Mech. Phys. Solids 55 1321-1339

[4] Kumar B, Feller J F, Castro M and Lu J 2010 Conductive bio-polymer nano-composites (CPC): chitosan-carbon nanotube transducers assembled via spray layer-by-layer for volatile organic compound sensing Talanta $\mathbf{8 1}$ 908-915

[5] Courty S, Mine J, Tajbakhsh A R and Terentjev E M 2003 Nematic elastomers with aligned carbon nanotubes: new electromechanical actuators Europhys. Lett. 64 654-660

[6] Tang X, Bansaruntip S, Nakayama N, Yenilmez E, Chang Y and Wang Q 2006 Carbon Nanotube DNA Sensor and Sensing Mechanism Nano Lett. 6 1632-1636

[7] Clayton L, Gerasimov T, Meyyappan M and Harmon J P 2005 Transparent PMMA/SWNT composites with increased dielectric constants Adv. Funct. Mater. 15 101-106

[8] Koohsorhi J, Abdi Y, Mohajerzadeh S, Hoseinzadegan H and Soleimani E A 2006 Novel self-defined field-emission transisotrs using carbon nano-tubes Carbon 44 2797-2803

[9] Frackowiak E and Béguin F 2002 Electrochemical storage of energy in carbon nanotubes and nanostructured carbons, Carbon 40 1775-1787

[10] Dai H, Hafner J H, Rinzler A G, Colbert D T and Smalley R E 1996 Nanotubes as nanoprobes in scanning probe microscopy, Nature 384 147-150

[11] Ramaratnam A and Jalili N 2004 Feasibility study of actuators and sensors using electroactive polymers reinforced with carbon nanotubes, Proceedings of SPIE 5385 349-356

[12] Carpi F, Gallone G, Galantini F and De Rossi D 2008 Enhancing The Dielectric Permittivity Of Elastomers in Dielectric Elastomers as Electromechanical Transducers Carpi F, De Rossi D, Kornbluh R, Pelrine R, Sommer-Larsen P eds., Amsterdam (Elsevier) 51-68 
[13] Zhang S, Zhang N, Huang C, Ren K and Zhang Q 2005 Microstructure and electromechanical properties of carbon nanotube/poly(vinylidene fluoride-trifluoroethylene-chlorofluoro-ethylene) compostes Adv. Mater. 17 1897-1901

[14] Pelrine R E, Kornbluh R D and Joseph J P 1998 Electrostriction of polymer dielectrics with compliant electrodes as a means of actuation Sensor Actuat. A-Phys 64 77-85

[15] Gallone G, Carpi F, Galantini F, De Rossi D and Levita G 2008 Enhancing the Electro-Mechanical Response of Maxwell Stress Actuators Adv. Sci. Tech. 61 46-531

[16] Opris D M, Molberg M, Walder C, Ko Y S, Fischer B and Nüesch F A 2011 New silicone composites for dielectric elastomer actuator applications in competition with acrylic foil Adv. Funct. Mater. 21 3531-3539

[17] Gallone G, Galantini F and Carpi F 2010 Perspectives for new dielectric elastomers with improved electromechanical actuation performance: Composites versus blend Polym. Int. 59 400-406

[18] Kussmaul B, Risse S, Kofod G, Waché R, Wegener M, McCarthy D N, Krüger H and Gerhard R 2011 Enhancement of dielectric permittivity and electromechanical response in silicone elastomers: Molecular grafting of organic dipoles to the macromolecular network Adv. Funct. Mater. 21 4589-4594

[19] Galantini F, Gallone G and Carpi F 2012 Effects of Corona treatment on electrical and mechanical properties of a porous dielectric elastomer IEEE T. Dielect. El. In. 19 1203-1207

[20] Vaisman L, Wagner H D and Marom G 2006 The role of surfactants in the dispersion of carbon nanotubes Adv. Colloid Interfac. Sci. 128-130 37-46

[21] Singh B P, Menchavez R, Takai C, Fuji M and Takahashi M 2005 Stability of dispersions of colloidal alumina particles in aqueous suspensions J. Colloid Interface Sci. 291 181-186

[22] Dyke C A and Tour J M 2004 Covalent functionalization of single-walled carbon nanotubes for materials applications J. Phys. Chem. A 108 11151-11159

[23] Dyke C A and Tour J M 2003 Solvent-free functionalization of carbon nanotubes J. Am. Chem. Soc. 125 1156-1157

[24] Mickelson E T, Chiang I W, Zimmerman J L, Boul P J, Lozano J, Liu J, Smalley R E, Hauge R H and Margrave J L 1999 Solvation of fluorinated single-wall carbon nanotubes in alcohol solvents J. Phys. Chem. B 103 4318-4322

[25] Ying Y, Saini R K, Liang F, Sadana A K and Billups W E 2003 Functionalization of carbon nanotubes by free radicals Org. Lett. 5 1471-1473

[26] Liang F, Sadana A K, Peera A, Chattopadhyay J, Gu Z, Hauge R H and Billups W E 2004 A convenient route to functionalized carbon nanotubes Nano Lett. 4 1257-1260

[27] Hong C-Y, You Y-Z, Pan C-Y 2006 Polymer 47 4300-4309

[28] Gallone G, Carpi F, De Rossi D, Levita G and Marchetti A 2007 Dielectric constant enhancement in a silicone elastomer filled with lead magnesium niobate-lead titanate Mat. Sci. Eng. C 27 110-116

[29] Gallone G, Carpi F, Galantini F, De Rossi D and Levita G 2008 Enhancing the Electro-Mechanical Response of Maxwell Stress Actuators Adv. Sci. Tech. 61 46-53

[30] Carpi F, Gallone G, Galantini F and De Rossi D 2008 Silicone-Poly(hexylthiophene) blends as elastomers with enhanced electromechanical transduction properties Adv. Funct. Mater. 18 235-241 